\title{
BASES PSYCHO-PHYSIOLOGIQUES DE LA DOULEUR
}

\author{
H. TuRNIER, M.D. ${ }^{\circ}$
}

LE TERME D'AFFECTIVITÉ, selon le Vocabulaire de Lalande, désigne "le caractère générique du plaisir, de la douleur, des émotions qu'on appelle du nom commun d'états affectifs." Plaisirs, douleurs, sentiments, passions sont des états affectifs. L'affectif est subjectif. Une joie, une passion, etc. sont des états éprouvés par un sujet.

Le plaisir et la douleur sont les tonalités de base de la vie affective. Ce sont des données immédiates de l'affectivité. Aussi est-il difficile de définir le plaisir et la douleur. La définition suppose l'analyse, la réduction en éléments simples. Or le plaisir et la douleur semblent des données élémentaires qu'il n'èst pas possible de réduire à quelque chose de plus simple.

D'autre part, définir c'est traduire en concepts, éclairer par des rapports objectufs. Or l'affectif est purement subjectif, purement "vécu." Le plaisir et la douleur se prêtent peu à l'expression conceptuelle, car ce ne sont pas des connaissances mais des expériences subjectives. Gide écrit dans les Nourritures terrestres: "Il m'est égal de lire que les sables des plages sont chauds, je veux que mes pieds nus le sentent." Dans cette perspective il n'y aurait rien à dire sur l'affectivité, il n'y aurait qu'à la vivre.

\section{ESSAI d'UNE CLASSIFICATION}

On distingue communément les plaisirs et les peines d'ordre physique et d'ordre moral. Lès premiers supposent une action directe d'un excitant sur l'organisme. Ils sont généralement localisables à un endroit précis du corps. Les secondes ont des causes plus complexes. Elles sont déterminées non par un excitant directement repérable mais par la signification d'ensemble que revêt une situation à l'égard de nos désirs. Ainsi Lavelle observait que la douleur physique est localisable dans l'espace (en tel lieu du corps) et vécue instant par instant, tandis que la souffrance porale est liée au temps: l'homme peut être torturé par des souvenırs (remords); il peut être inquiet pour l'avenir. Le souci, le remords, l'angoisse ne s'expliquent que par la pensée du passé et de l'avenir.

Cette distinction a été refusée. Ribot affirmait qu'il n'y avait pas de différence essentielle entre l'inquiétude métaphysique et le mal de dents. Selon lri, quelle que sort l'origine d'un état affectif, c'est toujours un état vécu par la conscience. D'autre part une souffrance "morale" peut avoir une origine purement organique. La tristesse du malade "mélancolique" comme l'euphorie du maniaque s'expliquent par un dérèglement du diencéphale (Delay'). Le langage courant ne rapproche-t-il pas volontiers peines et plaisirs physiques et moraux. On parlera d'une humiliation "cuisante," d'une vanité agréablement chatouillée par un compliment.

"Hôpital Sante Justne, Montreal, P.Q. 
Plaisirs et douleurs physiques dépendent eux aussi non seulement d'une excitation locale mais de la signification d'une situation d'ensemble. Tel coup de poing reçu au cours d'un match de boxe sera moins doulqureux que le même coup reçu à la suite d'une discussion non amicale. Dans le premier cas, le coup de poing a une signification sportive; dans le second cas, une signification "hostile." L'intensité d'une douleur est fonction de l'activité globale de l'organisme. C'est ainsi qu'il est possible "d'opérer éveillé un cheval qui mange de l'avoine."

Cette position èst loin d'être convaincante. L'èxpérience de chaque jour montre que la douleur purement physique et la souffrance morale-même si ces états sont souvent mêlés-sont malgré tout distinctes. La douleur physique et le plaisir physique sont plus étroitement lịes au corps.

La souffrance et les satisfactions morales échappent pour une part à lạ localisation spatiale et à l'instant. Mais il faut reconnnaître que la souffrance morale qui suppose l'imagination et le souvenir a d'ailleurs quelque chose de moins authentique que la douleur physique. Alain le montre lumineusement dans ses "Propos sur le bonheur." Une souffrance de limagination, attente pénible ou douloureux souvenir, "est bien près d'être une souffrance imaginaire. "La peur de la mort," disait Epicure, "est toujours un peu une comédie car tant je suis là, capable de concevoir et de sentir, la mort n'est pas encore présente, et quand elle sera là c'est moi qui n'y serai plus. Je souffre à la penșée que mon cadavre futur șera rongé par les vers. Mais le moment venu je n'en aurai pas conscience." Les pleurs que nous arrachent la douleur physique ont quelque chose de plus vrai que ceux qui viennent des souffrances morales. Comme l'écrit M. Thibon: "Il n'y a peut-être qu'une dpuleur vraie, sauf chez les saint's, c'est lá douleur physique." Et Chamfort s'écriait: "Mon Dieu, délivrez-moi des peines physiques, des morales je m’en charge."

Notre analyse se limitera donc tout d'abord aux plaisirs et aux douleurs physiques; parce qu'ils nous semblent les plus purs, les plus authentiquement affectifs. Mais comment éclairer rationnellement ce qui par essence est purement vécu, irrationnel et subjectif? Un moyen traditionnel de faire une théorie du plaisir et de la douleur consiste à introduire une dialectique, à les opposer l'un à l'autre. Entre les deux, il y aurait au moins cette relation logique de la contradiction.

\section{ThÉORJES SUR LA NATURE DU PLAISIR ET DE LA DOULEUR}

Lidée que le plaisir est absence de douleur se rencontre déjà chez Platon. Dans le Phédon, Socrate condamné à mort et libéré quelques instants de ses chaînes, éprouve un grand sentiment de soulagement, un véritable plaisir à faire aller librement bras et jambes alors qu'autrefois, quand il était libre, il ne savait pas goûter ce plaisir. Le plaisir se confond ici avec la cessation de la douleur infligée par les chaînes. Même opinion chez Epicure: le suprême plaisir, c'est l'absence d'agitation. Même opinion chez Schopenhauer pour qui.la souffrance seule est positive; le plaisir n'est que la négation je la suffrance.

Ces théories ne résistent pas à la critique. Elles sont toutes inspirées d'une conception d'ordre moral dévalorisant le plaisir afin d'interdire à l'homme sa recherche. 
En réalité l'absence de douleur et le plaisir sont deux états bien distincts. Le plaisir et la douleur appartiennent à des fonctions psycho-bidlogiques tout à fait différentes. Le plaisir est le couronnement d'une activité et la recherche du plaisir se confond avec l'appétit de vivre.

Alors que la plaisir, précédé par un besoin, suppose la conquête d'un objet .qui est d'abord absent, la douleur révèle au contraire la présence d'un obstacle et provoque un mouvement réflexe pour nous en délivrer. Le plaisir marque le succès de l'impulsion vers un objet, la douleur précède l'expulsion d'un obstacle.

Les physiologistes ont longtemps vu dans la douleur l'effet d'un excitant trop intense qui endommageait ou. détruisait un récepteur sensoriel. Mais à la suite des travaux de Von Trey on a pu sa dęmander si la douleur n'était pas un "sixième sens." Il semble bien que "le sens de la douleur" ait à sa disposition des récepteurs spécialisés (les points de douleur, qui à la surface de la peau se distinguent des points de pression et des points thermiques) prolongés par des fibres nerveuses spéciales, "fibres à faible diamètre, faiblement myéliniséés ou même totalement dépourvues de myéline' (par exemple les fibres grises de Remak du système nerveux viscéral sympathique), ces fibres spécialisées sont d'ailleurs à conduction très lente. Touchez du doigt un vase brûlant: vous éprouverez la sensation tactile du contact aviant d'éprouver la sensation douloureuse. Dans le cerveau, c'est le thalamus qui paraît contenir les centres de la douleur.

Jacquenoud pense que la sensibilité douloureuse varie beaucoup selon le tissu considéré. La sensibilité cutanée et sous-cutanés est sensiblement métamérique. La section d'une racine poștérieure ne détermine pas d'anesthésie car il y a une certaine interpénétration entre les métamères voisins. La clinique, les sections neuro-chirurgicales et la rachi-anesthésie permettent cependant une localisation assez précise de zones sensibles correspondantes aux nerfs rachidiens. Un principe identique est à la base de la sensibilité musculaire tandis que la sensibilité radiculaire viscérale est moins précise (Jacquenoud).

\section{Conciusion}

Puisque la douleur est liée à des dispositifs spécifiques, il faudrait admettre qu'elle possède une fonction biologique propre. Mais n'est-il pas paradoxal d'attribuer une fonction à cette sensation que tous les êtres vivants cherchent à éviter.

Aristote pourtant avait déjà parlé de la finalité du plaisir et de la douleur. Tandis que la plaisir exprime l'accomplissement d'une fonction, la douleur signale un danger à celư qui la ressent et ce signal peut permettre à l'être vivant de l'éviter ou de l'atténuer.

\section{INDEX BIBLIOGRAPHIQUE}

Alain. Propos du bonheur. Communication persomelle Von Trey.

Delay. Aspects de la psychiatrie modeme. Presses Universitaires de France.

Fulton, J. F. The Physiology of the Nervous System. Oxford University Press (1958).

GmE, A. Nourritures terrestres.

JACQUenoud. Encyclopédie Médico-chirurgicale 3G. 065 A10, La douleur, p. 3 (1939):

LALANDE. Vocabulaire, chap. 1, p. 1 (1925).

Lavelíle. Presses Universitaires de France. 
Lrvingstone, W. R. Mécanisme de la douleur. Macmillan Editeur, New-York et Londres (1947).

Monis, G. Physiologie du système neurveux central. Masson Editeur, Päris (1955)。

Penfield, W. \& Jasper. Epilepsy and the Functional Anatomy of the Human Brain, p. 54 (1954).

Rrвot. Vie inconsciente et mouvements, p. ix. Bibliothèque physiologie contemporaine (1960). Thibon \& Chamfort. Cours Professeur Claudius, Dr. en philosophie (1960). 